\title{
Active noise feedback control using a neural network*
}

\author{
Zhang Qizhi and Jia Yongle \\ Department of Automation, Beijing Institute of \\ Machinery, Beijing, 100085, P. R. China
}

Received 2 May 2000

Revised 7 September 2000

The active noise control (ANC) is discussed. Many digital ANC systems often based on the filter-x algorithm for finite impulse response (FIR) filter use adaptive filtering techniques. But if the primary noise path is nonlinear, the control system based on adaptive filter technology will be invalid. In this paper, an adaptive active nonlinear noise feedback control approach using a neural network is derived. The feedback control system drives a secondary signal to destructively interfere with the original noise to cut down the noise power. An on-line learning algorithm based on the error gradient descent method was proposed, and the local stability of closed loop system is proved using the discrete Lyapunov function. A nonlinear simulation example shows that the adaptive active noise feedback control method based on a neural network is very effective to the nonlinear noise control.

\section{Introduction}

Active noise control (ANC) is based on the simple physics principle of destructive interference of propagating acoustic waves. Active attenuation is a more attractive means to achieve large amounts of noise reduction in a small package or in a duct, particularly at low frequencies (bellow $500 \mathrm{~Hz}$ ). A relatively simple feedback control system for a duct is illustrated in Fig. 1. The ANC system uses the estimated primary acoustic noise signal to generate a signal $y(n)$ of equal amplitude but $180^{\circ}$ out of phase. This antinoise signal is used to drive the loudspeaker to produce a canceling sound that attenuates the primary acoustic noise traveling in the duct.

\footnotetext{
* Supported by Science Founds of Educational Committee of Beijing City (99KJ44).
}

Many digital ANC systems use adaptive filtering techniques, often based on the filter-x algorithm for finite impulse response (FIR) filter [3] because of its simplicity and its relatively low computational load. The linear digital feedback controller based on filter-x algorithm may not perform well in the case that nonlinearities are found in an ANC system. The use of a nonlinear feedback controller can improve the control performance on a system with a nonlinear behavior. The neural network feedback control is a perfect control technology for nonlinear systems, and has been used in various systems with nonlinearities [1]. For the active control of sound and vibration, the use of neural networks as nonlinear control structure has been reported [4], and multilayer perceptron neural networks were used to control nonlinear plants. This paper will focus on the active noise control problem of nonlinear response of an unknown primary acoustic path. A feedback control structure based on multilayer feedforward neural network is proposed. The stability of closed loop system is proved utilizing the discrete Lyapunov function. A digital simulating example with nonlinear primary noise path shows that the neural feedback control approach proposed in this paper performs better than feedback filter-x method does in dealing with a nonlinear control problem.

\section{Active noise feedback control using a neural network}

Please refer to Fig. 2 for the development of the algorithm for adapting a neural network based feedback controller. To make it simple, only the nonlinearity in the primary noise path is considered. A multilayer feedforward neural network based feedback controller is adopted. There is only one linear neuron in output layer. The neural network structure is shown in Fig. 3. The main symbols used in this paper are defined as follows:

$\mathbf{x}(k)=[x(k) x(k-1) \ldots x(k-I)]^{T} \quad x(k)$ is the estimated value of $d(k)$ at time $k$, 


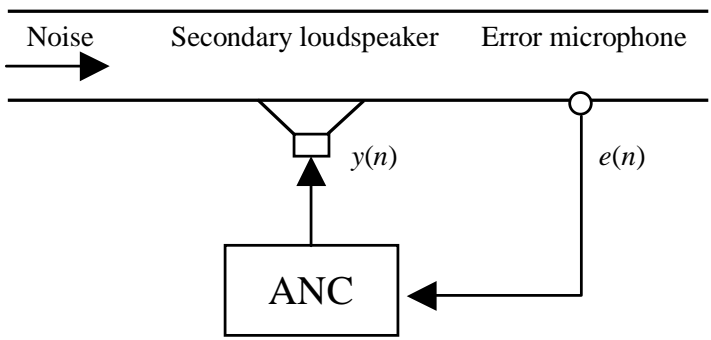

Fig. 1. Feedback control system in a duct.

$\mathbf{z}(k)=\left[z_{1}(k) z_{2}(k) \ldots z_{J}(k)\right]^{T} \quad z_{j}(k)$ is the output of the jth hidden neuron at time $k$,

$\mathbf{u}(k)=[u(k) u(k-1) \ldots u(k-n)]^{T} \quad u(k)$ is the output of the neural network at time $k$,

$n(k)$ the reference noise input at time $k$,

$y(k)$ output of secondary source at time $k$,

$d(k)$ the primary noise at time $k$,

$e(k)$ the input of error microphone at time $k$,

$\mathbf{h}=\left(h_{0} h_{1} \ldots h_{n}\right)$ the filter coefficients of secondary source path,

$\mathbf{c}=\left(c_{0} c_{1} \ldots c_{n}\right)$ the filter coefficients of estimated secondary source path,

$I, J$ number of the neurons in input layer and in hidden layer,

$W_{i j}^{I}(k)$ the connected weight from $i$ th input neuron to $j$ th hidden neuron,

$\mathbf{W}_{j}^{I}(k) \mathbf{W}^{I}(k)$ the weight row vector and matrix from input layer to hidden layer,

$\mathbf{W}^{O}(k)$ the weight row vector from hidden layer to output neuron,

$\mathbf{W}(k)$ the weight matrix combined $\mathbf{W}^{O}(k)$ and $\mathbf{W}^{I}(k)$,

$f_{j}(x)$ the activation function of $j$ th hidden neuron.

In the ideal case where the estimated secondary path $\mathbf{c}$ is equal to the real secondary path $\mathbf{h}$, then an equivalent feedforward architecture could be obtained [3]. But in general case, $\mathbf{c} \neq \mathbf{h}$, then the NN control system is a feedback control system.

Using the symbols we can obtain the outputs of hidden neurons, neural network and secondary source at time $k$ :

$$
\begin{aligned}
z_{j}(k) & =f_{j}\left(\sum_{i=0}^{I} W_{i j}^{I}(k) x(k-i)\right) \\
& =f_{j}\left(\mathbf{W}_{j}^{I}(k) \mathbf{x}(k)\right) \\
u(k) & =\sum_{j=0}^{J} W_{j}^{O}(k) z_{j}(k)=\mathbf{W}^{O}(k) \mathbf{z}(k)
\end{aligned}
$$

$$
y(k)=\sum_{j=0}^{n} h_{j} u(k-j)=\mathbf{h u}(k)
$$

The error function of system is defined as

$$
J(k)=e^{2}(k)=[y(k)+d(k)]^{2}
$$

The weights can be adjusted according to the gradient descent method

$$
\mathbf{W}(k+1)=\mathbf{W}(k)-\mu \frac{\partial J(k)}{\partial \mathbf{W}(k)}
$$

The gradient of error in (4) with respect to the weights is represented by

$$
\begin{aligned}
\frac{\partial J(k)}{\partial \mathbf{W}_{j}^{I}(k)} & =2 e(k) \frac{\partial y(k)}{\partial \mathbf{W}_{j}^{I}(k)} \\
& =2 e(k) \sum_{i=0}^{n} h_{i} \frac{\partial u(k-i)}{\partial W_{j}^{I}(k)} \\
\frac{\partial J(k)}{\partial \mathbf{W}^{O}(k)} & =2 e(k) \frac{\partial y(k)}{\partial \mathbf{W}^{O}(k)} \\
& =2 e(k) \sum_{i=0}^{n} h_{i} \frac{\partial u(k-i)}{\partial \mathbf{W}^{O}(k)} \\
\frac{\partial u(k)}{\partial \mathbf{W}^{O}(k)} & =\mathbf{z}(k) \\
\frac{\partial u(k)}{\partial \mathbf{W}_{j}^{I}(k)} & =\sum_{i=0}^{J} \frac{\partial u(k)}{\partial z_{i}(k)} \frac{\partial z_{j}(k)}{\partial \mathbf{W}_{j}^{I}(k)} \\
& =W_{j}^{O}(k) f_{j}^{\prime}\left(\mathbf{W}_{j}^{I}(k) \mathbf{x}(k)\right) \mathbf{x}(k)
\end{aligned}
$$

If the weights $\mathbf{W}(k)$ are made to adapt slowly enough with time [2], the gradients of $u$ in (6) and (7) can be approximately written as:

$$
\begin{aligned}
& \frac{\partial u(k-i)}{\partial \mathbf{W}^{O}(k)} \approx \frac{\partial u(k-i)}{\partial \mathbf{W}^{O}(k-i)} \\
& \frac{\partial u(k-i)}{\partial \mathbf{W}_{j}^{I}(k)} \approx \frac{\partial u(k-i)}{\partial \mathbf{W}_{j}^{I}(k-i)}
\end{aligned}
$$

So the gradient of error function with respect to the weights can be written as:

$$
\begin{aligned}
\frac{\partial J(k)}{\partial \mathbf{W}_{j}^{I}(k)}= & 2 e(k) \sum_{i=0}^{n} h_{i} W_{j}^{O}(k-i) f_{j}^{\prime} \\
& \left(\mathbf{W}_{j}^{I}(k-i) \mathbf{x}(k-i)\right) \mathbf{x}(k-i) \\
\frac{\partial J(k)}{\partial \mathbf{W}^{O}(k)}= & 2 e(k) \sum_{i=0}^{n} h_{i} \mathbf{z}(k-i)
\end{aligned}
$$

In conclusion, the weights of neural network can be on-line adjusted using the update rule (5) with gradients calculated by (12) and (13). 


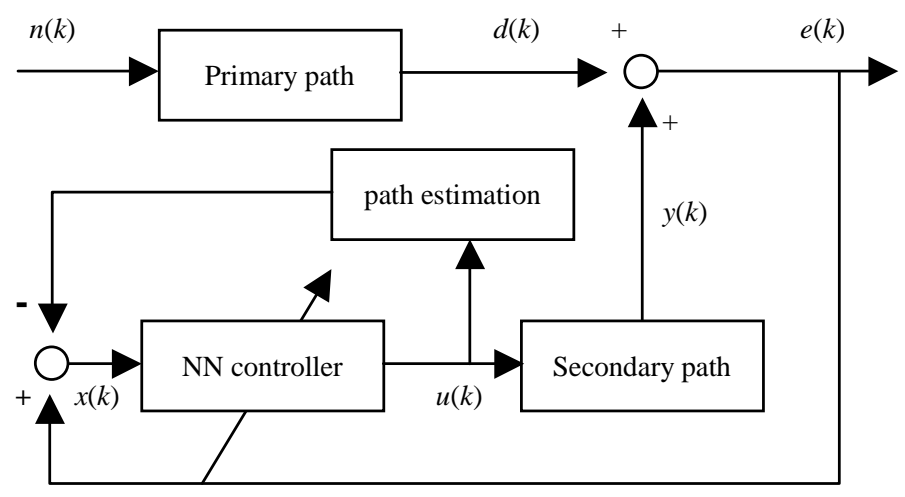

Fig. 2. Block diagram of NN based control system.

\section{The stability of active noise feedback control system}

The discrete-type Lyapunov function can be given by

$$
V(k)=\frac{1}{2} e^{2}(k)
$$

The change of the Lyapunov function due to the training process is obtained by

$$
\begin{aligned}
\Delta V(k) & =V(k+1)-V(k) \\
& =\frac{1}{2}\left[e^{2}(k+1)-e^{2}(k)\right]
\end{aligned}
$$

The error difference resulting from the learning can be represented by [1]

$$
\begin{aligned}
e(k+1) & =e(k)+\Delta e(k) \\
& =e(k)+\left[\frac{\partial e(k)}{\partial \mathbf{W}(k)}\right]^{T} \Delta \mathbf{W}(k)
\end{aligned}
$$

According to the update rule of the weights, we can obtain

$$
\begin{aligned}
\Delta \mathbf{W}(k) & =-2 \mu e(k) \sum_{i=0}^{n} h_{i} \frac{\partial u(k-i)}{\partial \mathbf{W}(k)} \\
& =-2 \mu e(k) \mathbf{h} \mathbf{G}(k)
\end{aligned}
$$

Where

$$
\begin{aligned}
& \mathbf{G}(k)=\frac{\partial \mathbf{u}(k)}{\partial \mathbf{W}(k)}, \\
& {\left[\frac{\partial e(k)}{\partial \mathbf{W}(k)}\right]^{T}=(\mathbf{h} \mathbf{G}(k))^{T}}
\end{aligned}
$$

A general stability theorem can be presented as follows:

Theorem: let $\mu$ be the learning rate for the weights of the neural network. We define $g_{\max }=\max _{k}\|\mathbf{G}(k)\|$, $g_{0}=\|\mathbf{h}\|$, where $\mathbf{G}(k)=\partial \mathbf{u}(k) / \partial \mathbf{W}(k),\|\bullet\|$ is the norm of matrix or vector. If the learning rate $\mu$ is chosen as $e<\mu<1 /\left(g_{0} g_{\max }\right)^{2}$, then the local stability of closed loop control system based on neural network is guaranteed.

Proof: define $\mathbf{g}(k)=\mathbf{h} \mathbf{G}(k)$, According to (15)(17), $\Delta V(k)$ can be represented as

$$
\begin{aligned}
\Delta V(k)= & \Delta e(k)[2 e(k)+\Delta e(k)] / 2 \\
= & -\left[\frac{\partial e(k)}{\partial \mathbf{W}(k)}\right]^{T} \mu e(k) \mathbf{h} \mathbf{G}(k) \\
& \left\{2 e(k)-2\left[\frac{\partial e(k)}{\partial \mathbf{W}(k)}\right]^{T} \mu e(k) \mathbf{h G}(k)\right\} \\
= & -\mu e(k)\|\mathbf{g}(k)\|^{2} \\
& \left\{2 e(k)-2 \mu e(k)\|\mathbf{g}(k)\|^{2}\right\} \\
= & -2 \lambda e^{2}(k)
\end{aligned}
$$

Since $\|\mathbf{g}(k)\| \leqslant\|\mathbf{h}\|\|\mathbf{G}(k)\| \leqslant g_{0} g_{\max }$, if the learning rate $\mu$ is chosen as $0<\mu<1 /\left(g_{0} g_{\max }\right)^{2}$, then $0<$ $\mu<1 /\|\mathbf{g}(\mathbf{k})\|^{2}$, so $\lambda=\mu\|\mathbf{g}(k)\|^{2}\left\{1-\mu\|\mathbf{g}(k)\|^{2}\right\}$ $>0$ and $\Delta V(k)<0$. Therefore the control system is local stable.

\section{Simulation results}

In this section, a comparison of illustrative results derived from the feedback filter- $x$ algorithm and a neural network based algorithm proposed in this paper is made. The acoustic paths were chosen as follows [2].

The primary acoustic path from noise source to error microphone

$$
\begin{aligned}
P(z)= & 0.05-0.001 z^{-1}+0.001 z^{-2}+0.8 z^{-3} \\
& +0.6 z^{-4}-0.2 z^{-5}-0.5 z^{-6}-0.1 z^{-7} \\
& +0.4 z^{-8}-0.05 z^{-9}
\end{aligned}
$$




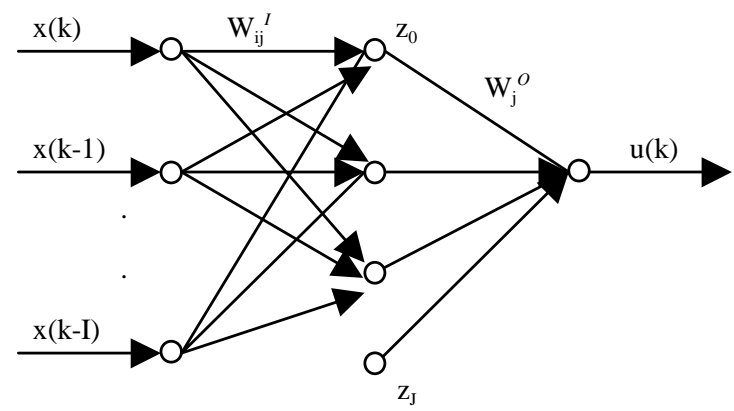

Fig. 3. Neural network structure.

The secondary acoustic path from secondary source to error microphone

$$
\begin{aligned}
H(z)= & 0.95-0.5 z^{-1}+0.1 z^{-2}+0.05 z^{-3} \\
& -0.005 z^{-4}
\end{aligned}
$$

The secondary feedback acoustic path from secondary source to reference microphone

$$
F(z)=0.6 z^{-1}
$$

Suppose there is nonlinearity in the primary acoustic path from noise source to error microphone. The disturbance

$$
\begin{aligned}
d(k)= & 0.05 x(k)-0.001 x(k-1)+0.001 x \\
& (k-2)+0.8 x(k-3)+0.6 x(k-4) \\
& -0.2 x(k-5)-0.5 x(k-6)-0.1 x \\
& (k-7)+0.4 x(k-8)-0.05 x(k-9) \\
& +x^{2}(k)
\end{aligned}
$$

The sampling frequency used for simulation was $1000 \mathrm{~Hz}$. The disturbance signal was chosen to be a $100 \mathrm{~Hz}$ pure tone signal and with an additional Gauss white noise signal. An off-line modeling technique [5] was used to estimate secondary acoustic path $H(z)$ during the training stage. At the end of training, the estimated model $C(z)$ will be fixed and used to on-line adjusting the parameter of controller in active noise control system. A 16-tap FIR filter was chosen in the filter-x algorithm. The number of neurons in the neural network controller was 6-6-1, it is selected according to some digital simulations, and the 6-6-1 architecture has the best control performance. In this digital experiment, the learning rate is 0.015 , and after about 1.5 second on-line learning, the training scheme converges. Figure 4 gives the simulating results of the canceling errors in the steady state in the frequency domain. The sound pressure level of error signal from

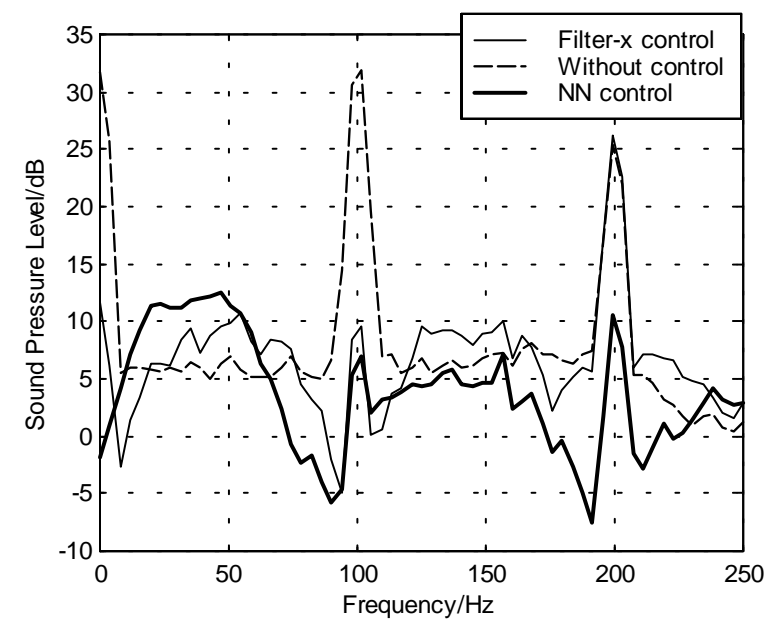

Fig. 4. Sound pressure level of the error signal.

the linear FIR controller trained with filter-x algorithm is shown in solid thin thread. The sound pressure level of error signal from the nonlinear neural network controller is shown in solid thick line. The dashed line shows the sound pressure level of disturbance signal when the ANC system turns off. It can be observed that the linear FIR controller does significantly reduce the $100 \mathrm{~Hz}$ tone (over $20 \mathrm{~dB}$ of reduction), but the tone at $200 \mathrm{~Hz}$ generated by the nonlinearity can not be reduced. The nonlinear neural network controller can reduce the $100 \mathrm{~Hz}$ tone (over $25 \mathrm{~dB}$ of reduction) and $200 \mathrm{~Hz}$ tone generated by the nonlinearity (over $15 \mathrm{~dB}$ of reduction).

\section{Conclusions}

The active noise control (ANC) is studied. If the primary noise path were nonlinear, the control systems based on adaptive filter technology that are often utilized in practice would be invalid. In this paper, an adaptive active nonlinear noise feedback control approach using a neural network is derived, a on-line learning algorithm based on the error gradient descent method is proposed, and local stability of closed loop system is proved based on the discrete Lyapunov function. A nonlinear simulation example shows that the adaptive active noise control method based on a neural network is very effective to the nonlinear noise control.

In this paper, only digital simulations are made, testing the NN controller by experiment will be the future work. 


\section{References}

[1] C.C. Ku and K.Y. Lee, Diagonal recurrent neural networks for dynamic systems control, IEEE Trans on neural networks $\mathbf{6}$ (1995), 144-156.

[2] H.C. David and W.S. Robert, Adaptive IIR filtered-v algorithms for ANC, J Acoust Soc Am. 101 (1997), 2097-2103.

[3] S.J. Elliott and P.A. Nelson, Active noise control, IEEE Signal
Process Mag 10 (1993), 12-35.

[4] B. Martin, P. Bruno and T.L.D. Chon, Improved training of neural networks for the nonlinear active control of sound and vibration, IEEE Trans. On Neural Networks 10 (1999), 391401.

[5] S.M. Kuo, I. Panahi and K.M. Chung, Design of active noise control systems with the TMS320 family, TI Application Report (1996), 18-19. 

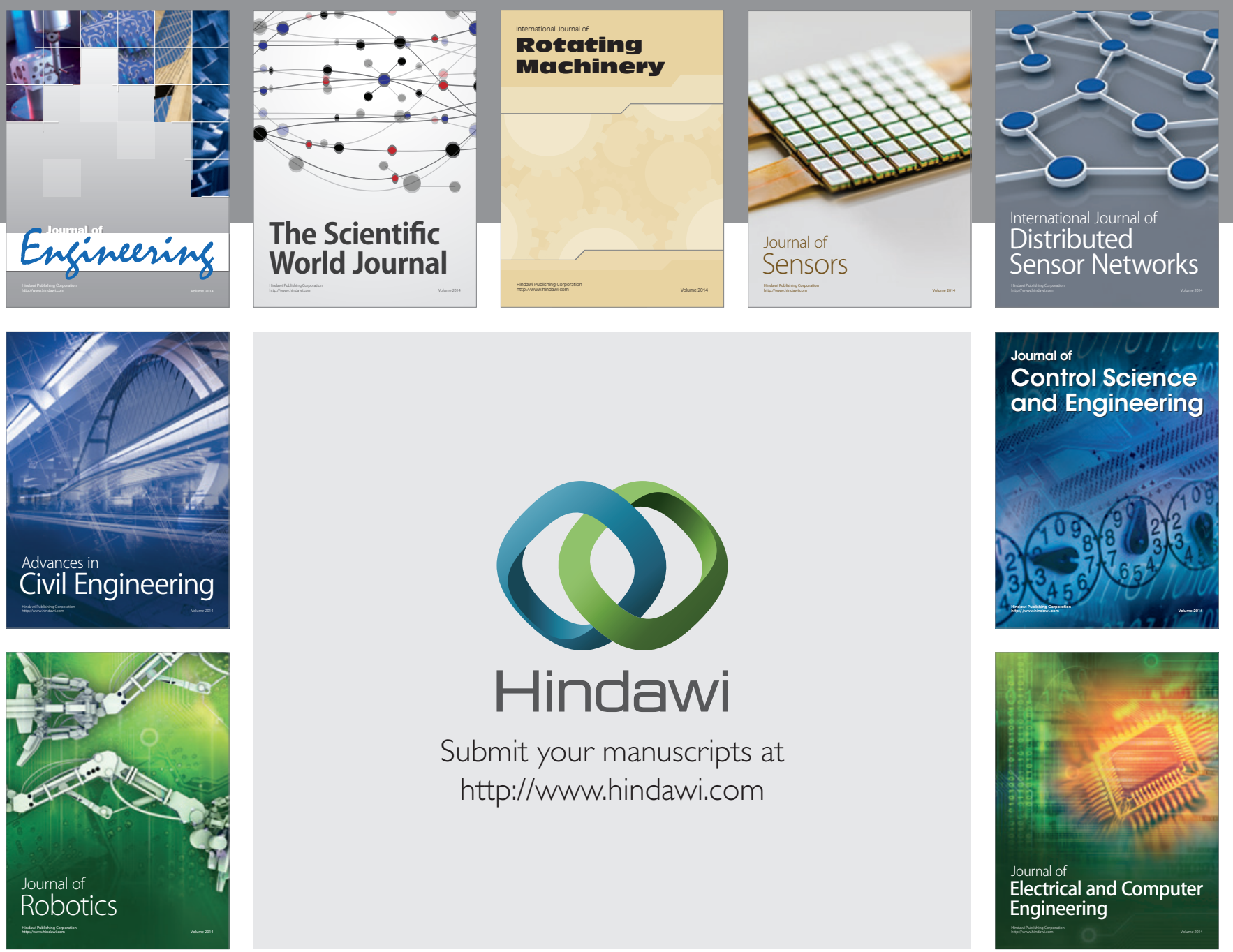

Submit your manuscripts at

http://www.hindawi.com
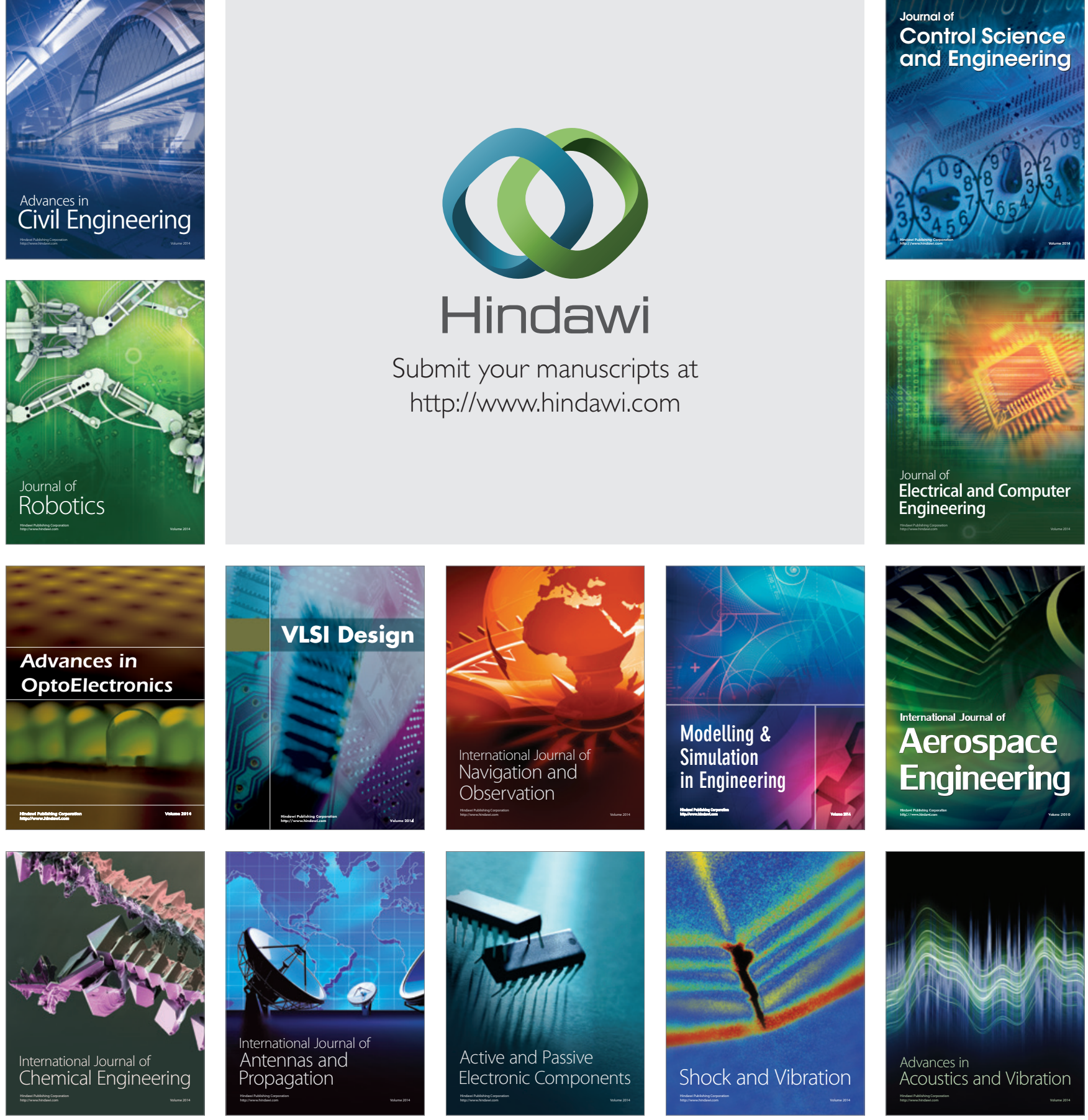